\section{Relación del valor de la clasificación y medición de la función motora gruesa (GMFM66-GMFCS) con episodios de dolor reportados por cuidadores en pacientes con insuficiencia motora de origen central (IMOC) \\ Relation between gross motor function measure and classification (GMFM66-GMFCS) and pain episodes reported by caregivers in patients with cerebral palsy}

Doris Valencia Valencia, Óscar Sotomonte Riveros

\section{RESUMEN}

Se investigó en pacientes de una institución de cuarto nivel (Fundación Hospital la Misericordia) especializada en pediatría, con diagnóstico de IMOC, quienes realizaron la prueba GMFM66; se procedió a entrenar a cuidadores en reconocer signos que pudiesen estar relacionados con la aparición de dolor vinculado a actividades diarias. Tras un periodo de observación se recolectaron los datos de dolor reportados por cuidadores para establecer la relación entre el nivel funcional de los pacientes y el dolor reportado. Se encontró un total de 28 pacientes aptos para la participación en el estudio con diferentes compromisos funcionales y se encontró una relación inversa entre GMFM66 y la escala LLANTO, y directa entre el GMFCS y la escala LLANTO. Esto que demuestra una relación entre un mayor compromiso funcional de los pacientes y la aparición de signos de dolor que reportasen los cuidadores, demostrando requerimiento de manejo integral del dolor en el paciente con discapacidad que no pueda comunicarse.

Palabras clave: parálisis cerebral, dolor, infantes con discapacidad.

\section{ABSTRACT}

The research, conducted in a $4^{\text {th }}$ level institution, specialized in pediatric care, with patients with cerebral palsy which took the GMFM66 test, then caregivers received training in identifying signs that could be related to pain associated with daily activities. After an observation period data was collected about pain signs noted by caregivers, so a relation between functional level and reported pain could be established. There were 28 patients who completed inclusion criteria, and an inverse relation between
Recibido:

25 de febrero de 2016

Aceptado:

25 de marzo de 2016

Autores:

Doris Valencia Valencia, MD

Fisiatra. Epidemióloga.

Departamento de Medicina Física

y Rehabilitación de la Universidad

Nacional de Colombia.

Óscar Sotomonte Riveros, MD

Fisiatra. Universidad El Bosque

Correspondencia:

dvalenciava@unal.edu.co

Conflictos de interés:

Ninguno 
GMFM66 and LLANTO score, and direct between GMFCS and LLANTO score was found, which shows the relation of lower functional levels with pain signs reported by caregivers, showing us the need of integral management an evaluation in patients with disability and unable to communicate.

Keywords: Cerebral palsy, Pain, Child with disability.

\section{INTRODUCCIÓN}

En el paciente con incapacidad motora de origen central (IMOC) el dolor tiene consecuencias que van desde la disminución de la adherencia a los tratamientos hasta la generación de experiencias negativas que pueden disminuir la disposición hacia el aprendizaje de las habilidades motoras $2,3,5,13$. Dichas habilidades motoras pueden ser evaluadas por medio de una escala de funcionalidad en los pacientes con IMOC llamada Gross Motor Function Classification System (GMFCS) ${ }^{6}$. Esta escala ha sido desarrollada en la universidad de McMaster, en el centro de investigación de discapacidad infantil, y clasifica a los pacientes en categorías de I hasta $\mathrm{V}$ de acuerdo con el nivel de autonomía que presentan para sus actividades y desplazamientos; cuanto más alta sea la puntuación (ej: GMFCS V), mayor será el compromiso motor y la discapacidad para los desplazamientos. También se desarrolló una medición de la función motora del paciente con calificación numérica, llamada Gross Motor Function Measure 66 $\left(\right.$ GMFM66) $^{6}$, la cual busca, de acuerdo con el desempeño motor del paciente, asignar un puntaje de su desempeño motor grueso durante la prueba, lo que permite tener una herramienta de seguimiento, facilitando así, en algunas ocasiones, establecer el pronóstico motor del paciente.

Es conocido que el dolor es una experiencia individual y ha hecho parte del desarrollo cultural en todas las sociedades ${ }^{3}$; cuenta con grandes componentes emocionales y orgáni$\cos ^{12}$, por lo que se convierte hoy día en uno de los principales adversarios en todos los procesos patológicos en cualquier sistema. Genera dudas y nubla el juicio de los pacientes y sus familiares, dejando muchas de sus manifestaciones sujetas a la experiencia individual de cada uno $^{13}$, por lo que se presenta una amplia subjetividad a la hora de ser evaluado. En los niños con discapacidad, de entrada hay una dificultad para lograr entablar una comunicación suficientemente buena como para saber si hay dolor y está presente como una barrera al desarrollo de las actividades del paciente ${ }^{12}$.

Así como existen medidas que pueden usarse para medir la discapacidad de los pacientes y medidas de valoración del dolor tales como la escala numérica análoga o visual análoga, existen escalas usadas para la medición del dolor en los pacientes no comunicantes, que se basan más en la observación del comportamiento de los pacientes para determinar la presencia de dolor; una de estas escalas es llamada LLAN. $\mathrm{TO}^{14}$, la cual, junto con otra llamada FLACC, evalúa múltiples variables y brinda una buena aproximación a la intensidad del dolor de los pacientes en función de una clasificación que va desde 0 a 10 según la intensidad del mis$\mathrm{mo}^{4}$, la cual podría utilizarse para realizar un abordaje terapéutico del dolor en los pacientes con discapacidad de manera más integral.

En la presente investigación se establece la relación entre diferentes valores de funcionalidad o discapacidad en los pacientes con IMOC y síntomas de dolor en los pacientes no comunicantes, con la meta de disminuir las barreras que existen para el tratamiento oportuno del dolor.

Se reclutaron pacientes del Hospital Fundación la Misericordia con diagnóstico de IMOC, quienes asistían a realizarse la prueba GMF66. Una vez seleccionados los pacientes que cumplían los criterios de inclusión, se procedió a entrenar a los cuidadores en reconocer signos que pudiesen estar en relación con la aparición de dolor vinculado a algunas actividades como 
la terapia física y las actividades de higiene personal. Posteriormente, tras un periodo de observación, se recolectaron los datos de dolor reportados por los cuidadores para establecer la relación entre el nivel funcional de los pacientes y el dolor reportado. Se encontró tras la recolección de datos, un total de 28 pacientes aptos para la participación en el estudio, con diferentes compromisos funcionales, y se encontró una relación inversa entre el valor del GMFCS y el GMFM66, así como entre GMFM66 y la escala LLANTO, y el GMFCS y la escala LLANTO. Esto demuestra una relación entre un mayor compromiso funcional motor de los pacientes y la aparición de signos de dolor que reportasen los cuidadores, reforzando el concepto de la necesidad de una valoración y manejo integral del dolor en el paciente con discapacidad que no pueda comunicarse, ya que es probable que muchos de estos pacientes no sean abordados para el tratamiento del dolor y esto puede ser un factor que entre en detrimento de los procesos de rehabilitación e integración del paciente.

\section{MATERIALES Y MÉTODO}

Tipo de investigación: Se realizó una investigación de tipo serie de casos prospectiva, en la que se estableció la relación entre el nivel de funcionalidad medido con GMFM66 y el dolor reportado por los cuidadores con la escala LLANTO y verificado con la escala FLACC, en niños con IMOC atendidos en la Fundación Hospital la Misericordia entre los meses de marzo y junio del 2015.

Población y muestra: La población de este estudio consistió en los pacientes con IMOC y su cuidador primario, quienes asistieron al servicio de medicina física y rehabilitación de la Fundación Hospital la Misericordia, en donde se realiza de forma regular el GMFM66. Fueron evaluados con escalas del paciente con IMOC en el contexto de discapacidad y dolor.

Criterios de inclusión: Pacientes entre los 2 y $\operatorname{los} 15$ años con diagnóstico de IMOC (diagnosticado por médico fisiatra o neurólogo pediatra), con las siguientes características:

- Incapacidad para comunicarse verbalmente de forma adecuada.

- Que mantengan estado de alerta ante los estímulos del medio.

- Que el cuidador primario se encuentre en condiciones de comprender y llevar a cabo las instrucciones para la recopilación de la información.

\section{CRITERIOS DE EXCLUSIÓN}

- Pacientes que como potenciales causas de dolor tengan otros problemas médicos diferentes de los asociados al IMOC, como procedimientos quirúrgicos recientes.

- Que puedan comunicarse verbalmente en forma clara y comprensible respecto a sus síntomas físicos.

- Pacientes que no reaccionen ante los estímulos del medio.

- Que no haya adecuada comprensión por parte del cuidador primario de los parámetros de las escalas usadas en la evaluación.

\section{ANÁLISIS DE RESULTADOS}

Los resultados obtenidos fueron analizados a través del paquete estadístico SPSS para el análisis de datos cuantitativos de los valores del GMFM66, GMSC, LLANTO y FLACC. A las variables numéricas se les aplicará prueba de normalidad (Shapiro-Wilk) por tratarse de una muestra inferior a 30 sujetos; dependiendo de los resultados, se aplicarán medidas de tendencia central; en el caso de no tener distribución normal, se obtendrán medidas de mediana y percentiles 25 y 75, buscando establecer relación entre: 
- Dolor por escala LLANTO y FLACC.

- GMFM66 y escala LLANTO en los ámbitos de terapia física, actividad lúdica e higiene.

- GMFCS y escala LLANTO en los ámbitos de terapia física, actividad lúdica e higiene.

- Presencia de manejo farmacológico analgésico.

- Presencia de manejo farmacológico para espasticidad.

- Presencia de manejo no farmacológico analgésico.

\section{RESULTADOS}

En total se obtuvieron datos completos y autorización por escrito para la evaluación de 28 pacientes; los pacientes que cumplían criterios de exclusión o para los cuales la información brindada por familiares fue incompleta o insuficiente para que la evolución se llevase a cabo a cabalidad no fueron incluidos en la base de datos. Se encontró en la distribución de edad de los pacientes una mediana de de 8.00, una moda de 3, que oscilaba entre los 2 y los 16 años; sin embargo, el $96,4 \%$ de los pacientes tenía 15 años o menos, con percentil 25 de 4 y un percentil 75 en 11,75; en su distribución se notó que los pacientes de 3 y 8 años eran los más presentes en el estudio y que su distribución es bimodal en el histograma, como se demuestra en la figura 1 y en la tabla 1 .

En los resultados del GMFCS se encontró que la mayoría de los pacientes estaban dentro de la clasificación funcional $\mathrm{V}$; es decir, presentan marcada limitación para el control del movimiento, sin tener posibilidad de buen control cefálico y de tronco, lo que hace que tengan dependencia total; estos corresponden al $57,1 \%$ de los pacientes. En los demás casos se encontraron pacientes con clasificación IV en
Tabla 1. Distribución de los casos por edad en la valoración del GMFM66.

\begin{tabular}{lcc}
\hline Edad & Frecuencia & Porcentaje \\
\hline 2 & 2 & 7,1 \\
3 & 4 & 14,3 \\
4 & 2 & 7,1 \\
5 & 1 & 3,6 \\
6 & 2 & 7,1 \\
8 & 4 & 14,3 \\
9 & 1 & 3,6 \\
10 & 2 & 7,1 \\
11 & 3 & 10,7 \\
12 & 1 & 3,6 \\
13 & 1 & 3,6 \\
14 & 2 & 7,1 \\
15 & 2 & 7,1 \\
16 & 1 & 3,6 \\
\hline Total & 28 & 100 \\
\hline
\end{tabular}

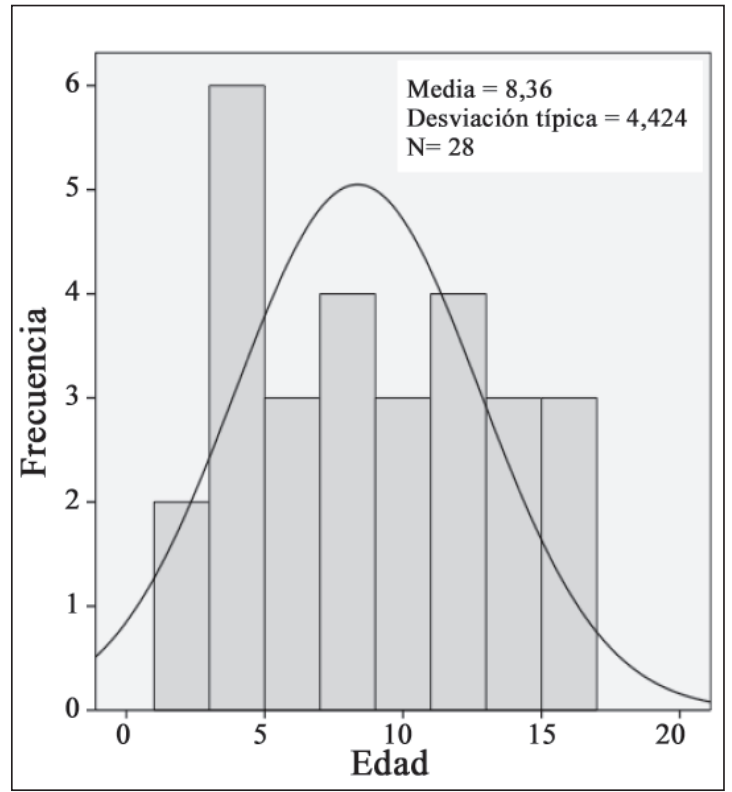

Figura 1. Histograma donde se muestra la distribución de los pacientes de acuerdo con la edad.

el $17,9 \%$, III en el 3,6\%, II en el $14,3 \%$ y I en el $7,1 \%$ de los pacientes, con una distribución sesgada en el histograma (tabla 2). En cuanto a los resultados realizados del GMFM66 se encontró una media de 38,20 puntos, con un valor mínimo de 0 y un máximo de 77 entre los pacientes evaluados. 
Tabla 2. Frecuencia de casos dentro de la clasificación GMFCS

\begin{tabular}{lcc}
\hline GMFCS & Frecuencia & Porcentaje \\
\hline 1 & 2 & 7,1 \\
2 & 4 & 14,3 \\
3 & 1 & 3,6 \\
4 & 5 & 17,9 \\
5 & 16 & 57,1 \\
\hline Total & 28 & 100,0 \\
\hline
\end{tabular}

Tratamientos de rehabilitación: Dentro de todo el grupo de pacientes evaluados, todos se encontraban realizando terapia física ocupacional y recibiendo intervención por fonoaudiología, a excepción de un paciente, quien solo recibía terapia física en casa, administrada por la cuidadora, ya que el paciente tenía dificultades para desplazarse con frecuencia a un centro de rehabilitación.

Consumo de analgésicos y uso de medidas no farmacológicas para el control del dolor: $\mathrm{Al}$ recolectar los datos se indagó respecto a las medidas adoptadas por los cuidadores para mitigar el dolor de los pacientes; se buscó si utilizaban analgesia farmacológica, ya fuera formulada o autoformulada, además de medidas no farmacológicas, principalmente medios físicos como medida del control del dolor. Se encontró uso de analgesia farmacológica de forma regular y formulada solo en el 10,7\% de los pacientes, mientras que el 53,6\% usaba analgesia autoformulada. En el $75 \%$ de los casos los cuidadores usaron medidas no farmacológicas para el control del dolor, destacándose el uso de calor y masajes para el control de los síntomas.

Espasticidad y manejo farmacológico de espasticidad: Del total de pacientes evaluados el $82,1 \%$ presentaba espasticidad dentro de su cuadro clínico, mientras que el 17,9\% restante presentaban alteraciones del tono diferentes de la espasticidad. Se documentó además que los pacientes que recibían tratamiento sistémico para espasticidad correspondían solamente al 28,6\% del total de los casos; se consideraron como manejo de espasticidad medicamentos estipulados en las guías de manejo de la Fundación Hospital la Misericordia; los utilizados en el grupo de pacientes fueron clonazepam, blaclofeno y tizanidina.

Evaluación del dolor: En el entorno de terapia física se revisaron las calificaciones del dolor según la escala LLANTO y FLACC, previo entrenamiento a los familiares en la observación de los elementos que corresponden a la escala, encontrándose dolor con valor de 0/10 (sin dolor) solo para el 14\% de los pacientes para ambas escalas y un 7\% para valor de 7/10 (el máximo valor encontrado) en escala LLANTO y un caso de 8/10 de dolor correspondiente a un 3,6\% de los pacientes a quienes se les realizó la escala FLACC. En cuanto a la distribución de los pacientes de acuerdo al nivel de dolor reportado con ambas escalas no se encontró una tendencia clara hacia determinado valor, debido a que se incluyeron pacientes con diferentes niveles funcionales y con compromisos variados con la distribución de casos mostrada en la figura 2. Adicionalmente, se evaluó la frecuencia del dolor en la actividad evaluada con parámetros de siempre, casi siempre, a veces o nunca, encontrándose que el 42,9\% de los casos presentaban signos de dolor siempre que se realizaba la intervención de terapia física, $28,6 \%$ casi siempre y $28,6 \%$ de los casos a veces.

Durante las actividades de higiene personal se notó que la mayoría de los pacientes no presentaban dolor, según lo documentado por escala FLACC y LLANTO; estos pacientes correspondieron al 67,9\% de los casos; en la valoración con ambas escalas se encontró un valor máximo de 6/10, el cual solo estuvo presente en un caso, correspondiente al 3,6\% para ambas escalas. Se encontró además que el 92,4\% de los pacientes presentó dolor leve, el cual se considera de una intensidad de 1/10 hasta 4/ 10. En las actividades lúdicas o actividades varias del diario vivir del paciente se encontró un comportamiento de los datos similar al de las actividades de aseo personal, lo que se podría explicar por la tendencia del paciente con 


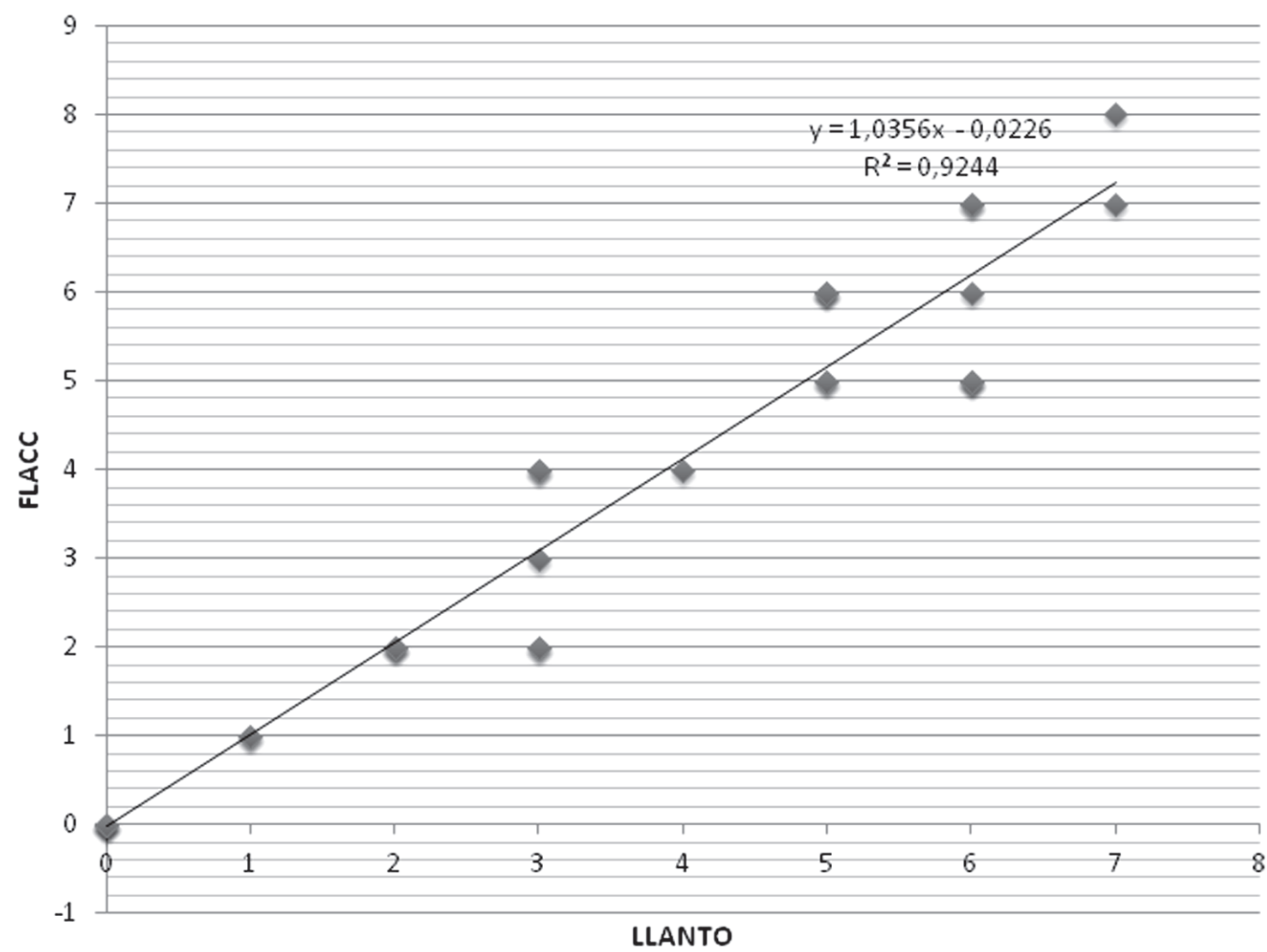

Figura 2. Gráfico de dispersión del resultado de la escala LLANTO y la escala FLACC, mostrándose relación linear de la misma.

compromiso funcional y espasticidad a permanecer continuamente en reposo, con una distribución demostrada en la tabla 3 . Adicionalmente, se evaluó la frecuencia de los signos encontrados en la actividad evaluada con parámetros de siempre, casi siempre, a veces o nunca, encontrándose que el $75 \%$ de los casos siempre presentaban los puntajes evaluados en escala FLACC y LLANTO $(67,9 \%$ de los pacientes no presentó signos de dolor durante esta actividad), el 3,6\% casi siempre y el $21,4 \%$ a veces.

Relación entre el nivel de discapacidad y dolor: En la evaluación de los sujetos se consideraron dos variables de funcionalidad, ambas en términos de función motora gruesa con el GMFCS y con el GMFM66, con los hallazgos descritos previamente en la descripción de la población. Lo que se encontró al realizar el análisis de correlación usando el coeficiente de
Tabla 3. Relación de resultados de la escala LLANTO vs. escala FLACC en el entorno de fisioterapia

\begin{tabular}{|c|c|c|c|c|c|c|c|}
\hline $\begin{array}{l}\text { FLACC } \\
\text { LLANTO } 0\end{array}$ & 1 & 2 & 3 & 4 & 5 & 6 & 7 \\
\hline 0 & 19 & & & & & & \\
\hline 1 & & 3 & & & & & \\
\hline 2 & & 1 & 1 & & & & \\
\hline 3 & & & & 1 & & & \\
\hline 4 & & & & & 1 & & \\
\hline 5 & & & & & & 1 & \\
\hline 6 & & & & & & & 1 \\
\hline
\end{tabular}

correlación de Spearman al establecer la relación entre GMFM66 y GMFCS, GMFCS y LLANTO, además de la relación entre GMFM66 y LLANTO fue:

1. Un coeficiente entre GMFM66 y GMFCS de $-0,866$, demostrando una correlación negativa fuerte entre elevados niveles de funcionalidad en las escalas GMFM66 
(cuanto más baja, mayor la discapacidad motora) y la escala GMFCS (cuanto más baja, mayor es la funcionalidad motora del paciente).

2. Un coeficiente entre GMFM66 y LLANTO de $-0,900$, demostrando una correlación negativa fuerte en relación con el nivel de discapacidad motora medido por GMFM66 (cuanto más bajo, mayor discapacidad motora) y la escala LLANTO (cuanto más alta, mayor es el dolor del paciente).

3. Un coeficiente entre GMFCS y LLANTO de 0,452 , demostrando una correlación de moderada a débil entre la clasificación por GMFCS (cuanto más alta, mayor el nivel de discapacidad) y la escala LLANTO (cuanto más alta, mayor es el dolor del paciente).

\section{DISCUSIÓN}

Al evaluar a los sujetos de la investigación se encontró un grupo heterogéneo de pacientes con diferentes niveles funcionales y diferentes niveles de dolor medido con las escalas mencionadas. Es importante tener en cuenta que si bien las herramientas de función motora gruesa como el GMFM66 o el sistema de clasificación GMFCS son muy útiles al contemplar la función motora de los pacientes, en cuanto a la medición objetiva del dolor de los pacientes aún no existe un gold standard para la medición del dolor en pacientes no comunicantes, por lo que se han usado diferentes escalas: una de las más conocidas es la escala FLACC, aunque en niños existe también la escala LLANTO, la cual, en el presente estudio, ha demostrado tener unos resultados muy similares a los de la escala FLACC en cuanto a la aproximación del dolor en pacientes pediátricos no comunicantes.

En la búsqueda de la relación entre las variables de medición del dolor por escala LLANTO y la función motora gruesa de los pacientes (GMFM66 y GMFCS), en las actividades de terapia física, se halló lo siguiente:
- Una fuerte correlación negativa entre los valores de GMFCS y GMFM66, lo que significa que los pacientes con una clasificación funcional motora con mayor compromiso (mayor nivel en la calificación, que significa mayor compromiso de la función motora gruesa) tendrán un peor desempeño en la medición de la función motora gruesa (menor puntuación significa mayor compromiso en función motora gruesa), lo que se manifiesta directamente en el abordaje de la discapacidad de estos pacientes, quienes a su vez tienen mayor riesgo de padecer condiciones potencialmente dolorosas como lo es la espasticidad, contracturas musculares y deformidades asociadas. Esta relación fue establecida por el grupo de investigación de McMaster, ya que las escalas de valoración GMFCS y GMF M66 fueron elaboradas simultáneamente, basándose en los mismos preceptos.

- Una fuerte correlación negativa entre los valores de GMFM66 y la escala LLAN$\mathrm{TO}$, demostrando la relación que existe entre peores desempeños en las pruebas de función motora gruesa (manifestado en bajos puntajes del GMFM66) y mayores puntuaciones en la valoración del dolor de acuerdo con la escala LLANTO (mayor puntuación implica mayores niveles de dolor reportados). Demostrando que sí existe una relación entre el nivel de discapacidad motora de los pacientes y el nivel de dolor que puedan presentar según los reportes por parte de los cuidadores, incluso pudiéndose contemplar la posibilidad de considerar siempre que se evalúe un paciente con una marcada discapacidad motora que este presentará signos de dolor que los padres puedan notar y reportar para poder realizar un enfoque más integral y temprano de los pacientes, en aras de mejorar su calidad de vida. Este hallazgo fue evidenciado por Barney y cols. en el año 20136, quienes encontraron al igual que se pueden utilizar como factor predictivo 
los niveles bajos de desempeño motor en estos pacientes para establecer el riesgo de presentar dolor.

- Una correlación positiva moderada entre la clasificación de la función motora gruesa por GMFCS y la escala LLANTO. Esto puede ser un resultado esperable si se tiene en cuenta que en comparación con el GMFM66, el GMFCS tiene muchas menos variables para tener en cuenta en la clasificación funcional de los pacientes (de I a V), lo cual puede permitir un rango muy amplio de pacientes dentro del mismo nivel en la clasificación, que a su vez significa que dentro el mismo nivel funcional puede haber pacientes con peor desempeño o con diferentes compromisos osteomusculares y neurológicos que potencialmente causen niveles diferentes de dolor. Así, esta correlación podría ser usada adecuadamente como una herramienta para predecir la posibilidad de los pacientes con discapacidad motora no comunicantes de padecer dolor, como factor asociado a su cuadro de IMOC en relación con los hallazgos de Barney y cols. en el año $2013^{6}$, quienes encuentran al igual que se pueden utilizar como factor predictivo los niveles bajos de desempeño motor en estos pacientes para establecer el riesgo de presentar dolor.

- Como hallazgo incidental, no corroborado estadísticamente por el bajo número de pacientes, se encontró que es posible que exista una relación entre el nivel de dolor reportado por los cuidadores y la administración de tratamiento sistémico para espasticidad, la cual es considerada como una de las principales causas de complicaciones osteomusculares y, por ende, de deformidad; sin embargo, se haría necesario más estudios para confirmar si el tratamiento para espasticidad, ya sea sistémico o local (no evaluado en este estudio), puede guardar relación con el nivel de dolor reportado por los cuidadores de los pacientes.

Durante el proceso de investigación se encontraron diferentes fortalezas en el estudio; una de ellas es la cantidad de pacientes que pudieron ser incluidos en el estudio, ya que con 28 sujetos de estudio la población evaluada se acerca a la cantidad de sujetos de estudio en investigaciones en otros países, permitiendo dejar un precedente en cuanto a la evaluación del dolor en pacientes pediátricos con discapacidad en Colombia, en donde aún hay muy pocos estudios en dolor pediátrico o en dolor y discapacidad. Otra de las fortalezas encontradas fue la del uso de diferentes escalas de valoración del dolor, lo cual permitió evaluar y verificar los hallazgos entre diferentes escalas, para poder utilizar la escala LLANTO como referente en esta investigación, lo que además permitirá en futuras investigaciones usar esta como referencia para la evaluación del dolor, como una herramienta usada en niños. Otra de las fortalezas encontradas en el presente estudio fue la de haber utilizado dos instrumentos para la evaluación de la función motora de los pacientes, tal como lo son el GMFM66 y la GMFCS, lo que abrió las posibilidades para evaluar ambas con sus ventajas o desventajas, como puede ser la facilidad para aplicarse (siendo más lenta de aplicarse la GMFM66, la cual puede tardear entre 30 y 40 minutos en su aplicación de acuerdo con la experticia del evaluador); adicionalmente, se permitió comparar las diferentes relaciones entre ambas escalas y el dolor reportado por los cuidadores.

Dentro de las debilidades encontradas durante el desarrollo de la investigación se encuentra la ausencia de un gold standard para la medición del dolor en los pacientes no comunicantes, lo que llevó al uso de otras medidas como son las de reporte por parte de los cuidadores por medio de las escalas LLANTO y FLACC. Otra de las debilidades es que el rango de edades de los pacientes evaluados fue muy amplio, y si bien esto permitió tener una población de estudio considerable, una distribución de edades como la que se encontró en el estudio no 
permite demostrar la relación que pueda haber entre la edad de los pacientes y la duración de su discapacidad junto con su exposición a entidades potencialmente dolorosas que puedan o no facilitar la percepción o manifestación de dolor. Adicionalmente a las debilidades ya presentadas, también se encuentra que existió un rango muy amplio de niveles de discapacidad incluidos entre los sujetos de estudio, lo que no permite establecer un punto de corte sobre el cual determinar desde qué nivel de discapacidad aumenta el riesgo de la percepción del dolor en los pacientes. Otra debilidad encontrada en el desarrollo de la investigación fue la de un número variable de casos en los cuales, pese a haber espasticidad, no había tratamiento sistémico para esta; además no se evaluó antecedente de manejo local de espasticidad o de otras medidas para el tratamiento de la misma, dejándose por fuera un factor que podría ser modificador del dolor en los pacientes no comunicantes con discapacidad. Sin embargo, este estudio deja un precedente para futuras investigaciones en donde se pueda contemplar la evaluación de los diferentes tratamientos para la espasticidad y el impacto que estos puedan tener en las manifestaciones de dolor en estos pacientes.

\section{CONCLUSIÓN}

El desarrollo de este estudio permitió ampliar la perspectiva en cuanto a la apreciación del dolor como uno de los elementos a considerar en la evaluación y manejo del niño con discapacidad, con dificultades en la comunicación. La correlación fuerte entre peores puntajes en GMFM66 y mayores niveles de dolor reportados por escala LLANTO confirma la sospecha de que los pacientes con mayores niveles de discapacidad y, por ende, con mayores posibilidades de presentar complicaciones osteomusculares tienen mayor riesgo de presentar dolor, y que adicionalmente se puede establecer como punto de partida la valoración de la función motora de los pacientes para considerar un abordaje integral del dolor en el paciente, y así evitar dificultades en los procesos de rehabilitación y en general evitar o controlar factores potencialmente asociados a una peor calidad de vida.

Ya demostrada por Barney y cols., la relación entre el nivel de discapacidad y el riesgo de percibir dolor permite establecer que en el abordaje de los pacientes con discapacidad motora de origen neurológico es necesario contemplar además de las medidas habituales de rehabilitación, una valoración del dolor, en lo posible facilitada por los cuidadores en el caso de que el autorreporte no sea posible. Esto con dos principales objetivos: uno de ellos realizar un manejo adecuado en aras de facilitar los procesos de rehabilitación y, por otro lado, aminorar la carga social y familiar en torno a los cuidados y en general de la rehabilitación de estos pacientes.

\section{REFERENCIAS}

1. Robaina G, Riesgo S, Robaina M. Definición y clasificación de la parálisis cerebral: ¿un problema ya resuelto? Revista de neurología 2007; 45(02): 110-117.

2. Alexander M, Mathews D. Pediatric rehabilitation: principles and practices Fourth edition. 4th edition. Demosmedical. 2009.

3. Hadden K, von Baeyer C. Pain in children with cerebral palsy: common triggers and expressive behaviors. PAIN 2002; 99: 281-288.

4. Reinoso F, Lahos A, Duran M, Caompo G, Castro L. Escala LLANTO: Instrumento español de dolor agudo en la edad preescolar. Anales de pediatría 2011; 74: 10-14.

5. Vervoort T, Trost Z, Van Ryckeghem D. Children's selective attention to pain and avoidance behavior: the role of child and parental catastrophizing about pain. PAIN 2013; 154: 1979-1988.

6. Barney C, Krach L, Rivard P, Belew J, Symon F. Motor function predicts parent-reported musculoskeletal pain in children with cerebral palsy. Pain reseach \& management 2013; 18(6): 323-327.

Relación del valor de la clasificación y medición de la función motora gruesa (GMFM66-GMFCS) con episodios de dolor reportados por cuidadores en pacientes con insuficiencia motora de origen central (IMOC) 
7. Von Baeyer C, Uman L, Chambers C, Gouthro A. Can we screen youn children to provide accurate self-reports of pain? PAIN 2011; 154: 1327-1333.

8. Boerner K, Chambers C, Craig K, Pillai R, Parker J. Caregiver accuracy in detecting deception in facial expressions of pain in children. PAIN 2013; 154: 525-533.

9. Young C, Sang H, Chin Y, Moon S, Kyoung $\mathrm{M}$, Ki H, Sung H, In H, In HC, Tae-jon C, Won J, Seung Y. Anterior Knee pain in patients with cerebral palsy. Clinics in orthopedic surgery 2014; 6: 426-431.

10. Mariani P, Buttaro M, Comba F, Zanotti E, Ali P, Piccaluga F. Bilateral simultaneous femoral neck fracture mimicking abdominal pain in a cerebral palsy patient. Case reports in orthopedics 2014. 925201.

11. Melanie P, Wen Y, Navneet B, Lauren S, Darcy F. Characteristics of pain in children and youth with cerebral palsy. Pediatrics 2013; 132 (2): 407-4013.

12. Miller C, Murphy M, Ineke M, Pitten C. Pain, anxiety and cooperativeness in children with cerebral palsy after rhizotomy: Changes throughout rehabilitation. Journal of pediatric psychology 1997; 22 (5): 698-705.

13. Engel J, Wilson S, Tran S, Jensen M, Ciol M. Pain catastrophizing in youths with physical disabilities and chronic pain.
Journal of pediatric psychology 2013; 38 (2): 192-201.

14. Berrin S, Malcarne V, Varni J, Burnwinkle T, Sherman S, Artavia K, Chambers H. Pain, fatigue, and school functioning in children with cerebral palsy. Journal of pediatric psychology 2007; 32(3): 330-337.

15. Parkinson K, Dickinson H, Arnaud K, Lyons A, Clover A. Pain in young people aged to 13 to 17 years with cerebral palsy: a crosssectional, multicentre European study. Archives in diseases of children 2013; 98 : 434-440.

16. Badia M, Riquelme I, Orgaz B, Acevedo R, Longo R, Montoya P. Pain, motor function and healtgh-related quality of life in children with cerebral palsy as reported by their physiotherapists. BMC pediatrics 2014; 14: 192.

17. Opheim A, Jahnsen R, Olsson E, Stanghelle J. Physical and mental components of healthrelated qualitu of life and musculoskeletal pain sites over seven years in adults with spastic cerebral palsy. Journal of rehabilita tion medicine 2011; 43: 382-387.

18. Hirsh A, Kratz A, Engel J. Survey results of pain treatments in adults with cerebral palsy. American journal of physical medicine and rehabilitation 2011; 90 (3): 207-216. 